\title{
Endothelial dysfunction and reduced heart rate variability in patients with metabolic syndrome
}

\author{
Elena Nikolaevna Smirnova, ${ }^{1,2}$ Evgeniya Alexandrovna Loran, ${ }^{1,2}$ Sofia Grigorievna Shulkina, ${ }^{1,2}$ \\ Sergey Yurievich Podtaev, ${ }^{1,2}$ Maxim Victorovich Trushin ${ }^{3}$ \\ ${ }^{1}$ Perm State Medical University named after Academician E.A. Vagner, Russian Ministry of Health, Perm; ${ }^{2}$ Institute of \\ Continuous Media Mechanics, Ural Branch of Russian Academy of Science, Perm; ${ }^{3}$ Kazan Federal University, Kazan, Russia
}

\begin{abstract}
According to experts of the World Health Organization (WHO), metabolic syndrome (MS) can be considered as pandemy of the XXI century, because its prevalence among the population of developed countries is about $25-35 \%$. In this study with the purpose of complex investigation of the autonomic nervous system and endothelial function we included 66 patients with MS between the ages of 25 and 61 (46.9 \pm 9.9 years). A comparison group of apparently healthy individuals (16 individuals, average age of $45.3 \pm 2.3$ years; $P>0.05$ ) was studied. To evaluate the response of microvascular tone, we used the method of wavelet analysis of skin temperature oscillations during cooling of the limb. All patients underwent the study of heart rate variability. The levels of insulin, endothelin-1, and vascular endothelial growth factor were determined using enzyme immunoassay. Patients with MS had significant differences in all metabolic parameters. Our study showed that in the group of MS there is a decrease of the variability of heart rhythm compared with the healthy group. Conducting cold test revealed signs of endothelial dysfunction in the MS group, which was manifested by the decrease of the index of vasodilation in the endothelial and neurogenic frequency range. In the study group we determined the increase in biochemical markers of endothelial dysfunction, which correlated with parameters of vasodilation. Also, the presence of endothelial dysfunction significantly correlated with signs of reduction of the variability of the heart rhythm.
\end{abstract}

\section{Introduction}

The prevalence of metabolic syndrome (MS) is growing in epidemic proportions. ${ }^{1}$ As it is known, the metabolic disorders as metabolic syndrome affect the functioning of all organs and systems; furthermore,

Correspondence: Maxim Victorovich Trushin, Kazan Federal University, Kazan, Russia.

E-mail: mtrushin@mail.ru

Key words: Metabolic syndrome; endothelial dysfunction; cold test; heart rate variability.

Funding: this work was supported by Grant RSF 14-15-00809.

Conflict of interest: the authors declare no potential conflict of interest.

Received for publication: 7 February 2017.

Revision received: 16 October 2017.

Accepted for publication: 20 October 2017.

This work is licensed under a Creative Commons Attribution NonCommercial 4.0 License (CC BY-NC 4.0).

(C) Copyright E.N. Smirnova et al., 2018

Licensee PAGEPress, Italy

Italian Journal of Medicine 2018; 12:51-56

doi:10.4081/itjm.2018.847 the most serious diseases develop in the cardiovascular system. Cardiovascular changes in MS manifest as arterial hypertension (AH), autonomic and endothelial dysfunction, which contribute to decrease the adaptive and reserve opportunities of an organism. ${ }^{2}$ Activation of the sympathetic nervous system contributes to the development of peripheral insulin resistance and plays an important role in the pathogenesis of these changes. In turn, hyperinsulinemia stimulates the sympathetic nervous system and increases the production of catecholamines, hence there is a need to fill the growing metabolic needs of the body. ${ }^{3}$

Currently, it has been identified the significant correlation between the decrease of the variability of the autonomic nervous system (ANS) and mortality from cardiovascular disease. ${ }^{4}$ Endothelial dysfunction is inextricably linked to insulin resistance through the stimulatory effects of insulin and the production of $\mathrm{NO}$ in the endothelium. The autonomic nervous system has a direct effect on the endothelium through $\alpha 2$ - and $\beta$ adrenergic receptors located on endothelial cells. ${ }^{5}$ For the evaluation of endothelial function biochemical markers are used, such as von Willebrand factor, vascular endothelial growth factor (VEGF), endothelin-1 (ET-1) and others. However, functional changes in the endothelium precede morphological changes in the vascular wall and vasomotor form of endothelial dysfunction often manifests itself by changes in vasodilatory reactions. ${ }^{6} \mathrm{~A}$ method of wavelet analysis of skin 
temperature oscillations is proposed as an instrumental non-invasive method. ${ }^{7}$

This study was aimed to investigate the endothelial function in patients with MS by using biochemical markers of endothelial dysfunction and instrumental methods of wavelet analysis of skin temperature oscillations and to identify the relationships between them. We also planned to study the heart rate variability (HRV) in patients with MS and to establish the relationship of the functional state of the endothelium and heart rate variability in patients with MS.

\section{Materials and Methods}

Our study included 66 patients with MS. MS was stated in accordance with the criteria of the International Diabetes Federation (IDF) (2005): abdominal obesity - waist circumference $\geq 94 \mathrm{~cm}$ in men, $\geq 80 \mathrm{~cm}$ in women in combination with any 2 of the following criteria - the increase of triglycerides (TG) $\geq 1.7$ $\mathrm{mmol} / \mathrm{L}$; decrease in low high-density lipoproteins (HDL) in women $<1.3 \mathrm{mmol} / \mathrm{L}$, in men $<1.03$ $\mathrm{mmol} / \mathrm{L}$; blood pressure $\geq 135 / 85 \mathrm{mmHg}$, increase in plasma glucose $\geq 5.6 \mathrm{mmol} / \mathrm{L}$. The study did not include patients receiving $\beta$-blockers and statins, with secondary forms of $\mathrm{AH}$, diabetes mellitus, coronary artery disease, cardiac arrhythmias, heart failure functional class 3-4, inflammatory diseases of the myocardium, systemic connective tissue diseases, and cancer. The levels of lipids, plasma glucose, creatinine, uric acid, transaminases, bilirubin were determined by standard biochemical methods. Insulin resistance was determined by the HOMA-IR index. Glomerular filtration rate (GFR) was calculated according to the CKD-EPI formula.

To study the level of endothelin-1, we used enzyme immunoassay Biomedica ENDOTELIN (1-21) measuring range $0.02-10 \mathrm{fmol} / \mathrm{mL}$. The concentration of the VEGF in the blood serum was determined by enzyme immunoassay using appropriate kits JSC Vector-best (Novosibirsk) photometer Stat Fax (Awareness Technology Inc., USA).

The heart rate variability was studied on computer electrocardiograph poly-spectrum rhythm and was processed using the software Poly-Spectrum NET. Before the analysis of HRV, patients were in a horizontal position at rest for $20 \mathrm{~min}$. The initial tone was determined by spectral and temporal analysis. Background recording recorded for $5 \mathrm{~min}$, then was assessed autonomic reactivity - according to active orthostatic test (AOP) for 6 min with the determination of blood pressure.

The initial tone is determined according to a spectral and temporal analysis of HRV in 5-min background recording, autonomic reactivity - by means of the AOP. We estimated parameters of the total power spectrum, an indicator of high frequency (HF) and low frequency (LF) oscillations; the intensity of very low frequency (VLF) oscillations, characterizing the impact of higher autonomic centers on subcortical vasomotor center, $\mathrm{LF} / \mathrm{HF}$ (vagosympathetic balance ratio is the ratio of the power waves of LF power to HF waves). Autonomic reactivity of the parasympathetic division of the ANS was determined by the coefficient K30:15, the sympathetic division - using the increase in the index LF/HF and by the dynamics of waves of LF and VLF range in the AOP. The condition of segmental (LF, HF) and suprasegmental (VLF) mechanisms of $\mathrm{HRV}$ was estimated by the index of centralization $(\mathrm{IC}) \mathrm{IC}=(\mathrm{HF}+\mathrm{LF}) / \mathrm{VLF}$. Actually, it is a quantitative characterization of the relationships between influences of central and autonomic contours of heart rate regulation. We used the following time parameters recommended for practical use: RRNN mean of all R-R intervals in the sample, SDNN, MS - standard deviation from the mean duration of $\mathrm{NN}$-intervals, PNN50 - the percentage representation of episodes of differences in successive intervals more than $50 \mathrm{~ms}$, coefficient of variation $(\mathrm{CV}) \%$.

To evaluate the response of microvascular tone, we used the method of wavelet analysis of skin temperature oscillations (WAST) during cooling of the contralateral limb. Time-frequency analysis of temperature fluctuations was performed using wavelet analysis. For the reconstruction of the oscillations in endothelial (0.02-0.0095 Hz), neurogenic (0.05-0.02 $\mathrm{Hz}$ ) and myogenic $(0.05$ to $0.14 \mathrm{~Hz}$ ) frequency bands we applied inverse wavelet transform. ${ }^{7}$

To assess the contribution of different mechanisms of regulation of vascular tone we selected the RMS value of oscillation amplitudes of skin temperature $(\Delta T)$ in the relevant frequency range. In each frequency band we estimated variations of the mean square vibration amplitudes: $\mathrm{k}=\mathrm{STi} / \mathrm{ST}_{1}$, where $\mathrm{ST}_{1}$ is the RMS amplitude of oscillation before a cold test, and STi is the RMS vibration amplitude for the corresponding time intervals (during the trial, for 3 and 10 min after the test). The obtained data are designated as an index of vasoconstriction and vasodilation. The index of vasoconstriction (IVC) is set to $\mathrm{k}=\mathrm{ST}_{2} / \mathrm{ST}_{1}$, where $\mathrm{ST}_{2}$ is the amplitude of the vibration during cold exposure. The index post-cold vasodilation -1 (IPV 1) is set to $\mathrm{k}=\mathrm{ST}_{3} / \mathrm{ST}_{1}$, where $\mathrm{ST}_{3}$ - amplitude of fluctuations during the first $3 \mathrm{~min}$ after cold exposure. The index post-cold vasodilation $-2(\mathrm{IPV}-2)$ is set to $\mathrm{k}=\mathrm{ST}_{4} / \mathrm{ST}_{1}$, where $\mathrm{ST}_{4}$ is the amplitude of oscillation in the range of 3 to $10 \mathrm{~min}$ after the cold test.

Statistical analysis of obtained data was carried out using the integrated package for statistical analysis Statistica 6. Assessment of the reliability of differences $(\mathrm{P})$ between groups was performed using nonparametric methods, comparison of qualitative and 
quantitative traits (Mann-Whitney criterion). For correlation analysis we used the Spearman rank correlation (R). Values are represented as median $(25 ; 75 \%$ percentile), and the average with standard deviation. Differences in the indices were considered significant at a significance level of $\mathrm{P} \leq 0.05$.

\section{Results}

For the purpose of complex study of the autonomic nervous system and endothelial function 66 patients with MS aged 25 to 61 years ( $46.9 \pm 9.9$ years) were included. As a comparison group we studied apparently healthy individuals (16 men, average age of $45.3 \pm 2.3$ years; $\mathrm{P}>0.05$ ).

The first degree of obesity was most prevalent in patients MS; $47 \%$, and 33\% had the second and $20 \%$ third degree of obesity. In $73 \%$ it was revealed violation of glucose tolerance, and $91 \%$ of patients had hypertension. The metabolic status of patients of the main group was characterized by elevated levels of cholesterol, TG, low-density lipoprotein, glucose, uric acid, insulin, glycated hemoglobin $\left(\mathrm{H}_{\mathrm{B}} \mathrm{A} 1 \mathrm{c}\right)$, HOMA index, and GFR was significantly lower than in the control group (Table 1).

Insulin levels were correlated with systolic $(\mathrm{r}=0.32 ; \mathrm{P}=0.01)$ and diastolic $(\mathrm{r}=0.27 ; \mathrm{P}=0.03)$ blood pressure levels, TG $(\mathrm{r}=0.32 ; \mathrm{P}=0.014)$ and uric acid $(\mathrm{r}=0.42 ; \mathrm{P}=0.005)$. The HOMA index was also correlated with uric acid $(\mathrm{r}=0.35 ; \mathrm{P}=0.04)$. The concentrations of the VEGF, endothelin-1 were significantly higher in MS patients (Table 2). Level VEGF correlated with the value of endothelin- $1(\mathrm{r}=0.43$, $\mathrm{P}=0.009$ ).

Analysis of initial autonomic status showed that in the comparison group dominated patients with eutonia $(48 \%)$ while in the MS group we primarily determined persons with sympathetic tone $(51 \%) \quad\left(\chi^{2}=4.28\right.$, $\mathrm{P}=0.01$ ) (Figure 1).

In background recording if patients with metabolic syndrome, we showed a reduction in the total power

Table 1. Clinical characteristics of patients.

\begin{tabular}{|c|c|c|c|}
\hline Parameters & Metabolic syndrome $(n=66)$ & Controls $(n=16)$ & $\mathbf{P}$ \\
\hline Age, years & $46.9 \pm 9.9$ & $45.3 \pm 2.3$ & 0.8 \\
\hline Females \% & $45 \%$ & $43 \%$ & 0.7 \\
\hline Waist circumference, $\mathrm{cm}$ & $109.8 \pm 15.2$ & $80.7 \pm 1.1$ & 0.0001 \\
\hline Body mass index, $\mathrm{kg} / \mathrm{m}^{2}$ & $35.7 \pm 5.1$ & $27 \pm 1.3$ & 0.0001 \\
\hline Systolic arterial pressure, $\mathrm{mmHg}$ & $145.6 \pm 16.2$ & $125.3 \pm 3.2$ & 0.0001 \\
\hline Diastolic arterial pressure, $\mathrm{mmHg}$ & $92.0 \pm 15.6$ & $78.5 \pm 3.3$ & 0.0001 \\
\hline Glucose (mmol/L) & $5.8 \pm 0.8$ & $4.21 \pm 0.12$ & 0.0001 \\
\hline $\mathrm{TC}(\mathrm{mmol} / \mathrm{L})$ & $5.8 \pm 0.13$ & $4 \pm 0.12$ & 0.004 \\
\hline HDL (mmol/L) & $1.3 \pm 0.34$ & $1.4 \pm 0.04$ & 0.3 \\
\hline $\mathrm{TG}(\mathrm{mmol} / \mathrm{L})$ & $2.1 \pm 0.7$ & $0.57 \pm 0.08$ & 0.001 \\
\hline $\mathrm{H}_{\mathrm{B}} \mathrm{A} 1 \mathrm{c} \%$ & $5.9 \pm 0.4$ & $5.1 \pm 0.07$ & 0.04 \\
\hline Uric acid (mmol/L) & $376 \pm 98.1$ & $220 \pm 23.1$ & 0.004 \\
\hline Insulin, mcME/mL & $17.6 \pm 10.2$ & $6.7 \pm 1.2$ & 0.005 \\
\hline HOMA-IR, units & $5.2 \pm 3.4$ & $1.25 \pm 0.7$ & 0.003 \\
\hline $\mathrm{GFR} \mathrm{mL} / \mathrm{min} / 1.73 \mathrm{~m}^{2}$ & $86.5 \pm 6.7$ & $112.7 \pm 7.6$ & 0.001 \\
\hline
\end{tabular}

TC, total cholesterol; HDL, high-density lipoproteins; TG, triglycerides; $\mathrm{H}_{\mathrm{B}} \mathrm{Alc}$, glycated hemoglobin; GFR, glomerular filtration rate.

Table 2. Levels of endothelin-1 and vascular endothelial growth factor.

\begin{tabular}{lccc}
\hline Parameter & \multicolumn{3}{c}{ Median values (25; 75\% percentile) } \\
\hline & Metabolic syndrome (n=66) & Controls (n=16) & P \\
\hline VEGF, picog/mL & $164(96-288)$ & $75(0-96)$ & 0.02 \\
\hline Endothelin-1 (units) & $0.5(0.3-2.2)$ & $0.3(0.1-0.5)$ & 0.017 \\
\hline
\end{tabular}

VEGF, vascular endothelial growth factor. 
spectrum $\left(\mathrm{TP}^{2}\right)$ (Table 3$)$. Indicators time analyses SDNN and CV in the group of metabolic syndrome was significantly lower. This indicates a decrease of the heart rate variability. The indicators characterizing the activity of the parasympathetic nervous system, RMSSD and pNN50\%, also had a tendency to decrease in the MS group $(\mathrm{P}=0.07)$.

The results of the active orthostatic test showed a decrease in TR in both groups. Reactivity of the sympathetic and parasympathetic systems did not differ in groups. We found an increase in index vago-sympathetic balance in both groups. But the increase in MS was insufficient and was below the comparison group ( $2.8 \mathrm{~m}$ and 3.6, $\mathrm{P}<0.05$ ).

Indices of endothelial function, obtained during the cold test are shown in Table 4. IVC reflects vascular reaction to cold exposure. Indexes post-cold of vasodilatation (IPV -1 and -2 ) reflect the activity vasodilatory mechanisms after cold exposure. In our case, in the comparison group IVC reaches a value of 0.47 , and the elimination of cold exposure is an increase in the index IPV IPV -1 and -2 to 1 , almost doubles. Thus, an adequate response of the endothelium to cold stress is characterized by a reduction in

Table 3. Values from rhythmograms.

\begin{tabular}{|c|c|c|c|c|}
\hline \multirow[t]{2}{*}{ Value } & \multicolumn{2}{|c|}{ Background recording } & \multicolumn{2}{|c|}{ Orthostatic test } \\
\hline & Control & MS & Control & MS \\
\hline $\mathrm{TPMS}^{2}$ & $3.438 \pm 2.682$ & $2026.9 \pm 1806.6^{*}$ & $3.376 \pm 2.109$ & $1689 \pm 1345^{*}$ \\
\hline$\% \mathrm{VLF}$ & $40.5 \pm 23.8$ & $41.8 \pm 16.2$ & $39.2 \pm 15.7$ & $47.9 \pm 14.3^{*}$ \\
\hline$\%$ LF & $29.4 \pm 13.6$ & $30.6 \pm 9.2$ & $49.9 \pm 14.5$ & $37.5 \pm 12.8^{*}$ \\
\hline$\% \mathrm{HF}$ & $30.1 \pm 19.8$ & $27.6 \pm 13.8$ & $10.9 \pm 4.2$ & $14.2 \pm 9.3$ \\
\hline $\mathrm{LF} / \mathrm{HF}$ & $1.6 \pm 1.9$ & $1.4 \pm 1.0$ & $5.8 \pm 2.8$ & $4.0 \pm 3.2 *$ \\
\hline IC & $1.5 \pm 0.8$ & $1.8 \pm 1.3$ & $1.9 \pm 1.0$ & $1.3 \pm 0.8^{*}$ \\
\hline SDNN & $54.4 \pm 23.2$ & $40.2 \pm 17.5^{*}$ & $55 \pm 17.1$ & $37.2 \pm 14.5^{*}$ \\
\hline RMSSD & $39.3 \pm 26.3$ & $30.3 \pm 21.9$ & $28.4 \pm 15.6$ & $17.6 \pm 8.9$ \\
\hline $\mathrm{pNN} 50 \%$ & $17.2 \pm 20.3$ & $10.3 \pm 15.3$ & $6.9 \pm 9.6$ & $2.6 \pm 4.6^{*}$ \\
\hline $\mathrm{CV} \%$ & $6.0 \pm 2.2$ & $4.4 \pm 1.5^{*}$ & $7.3 \pm 1.8$ & $4.8 \pm 1.6^{*}$ \\
\hline K30/15 & - & - & $1.26 \pm 0.15$ & $1.21 \pm 0.13$ \\
\hline
\end{tabular}

MS, metabolic syndrome; TP, total power spectrum; VLF, very low frequency; HF, high frequency; IC, index of centralization; $\mathrm{CV}$, coefficient of variation. $* \mathrm{P}<0.05$ between the groups, in the appropriate ranges.

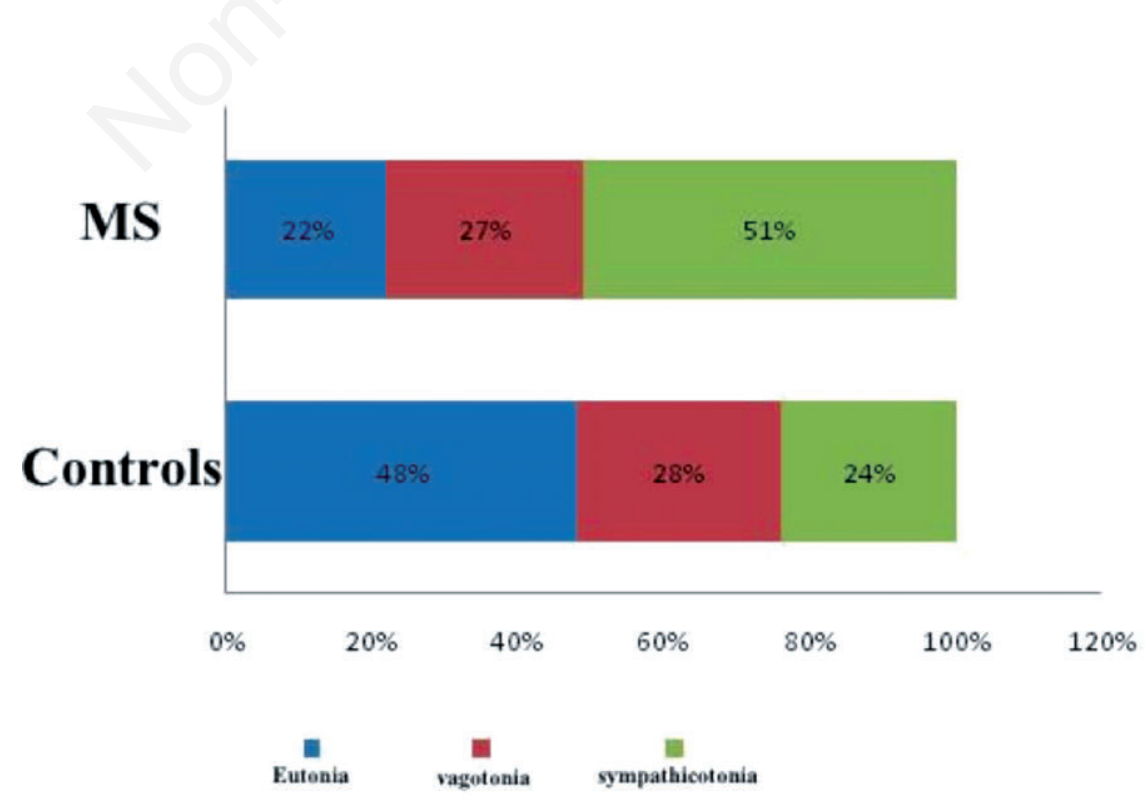

Figure 1. The distribution of patients in groups for initial vegetative tone. MS, metabolic syndrome. 
oscillation amplitudes, and then restoring their size.

Microcirculation disorders were revealed in the endothelial frequency range and partly in neurogenic one. In the group of patients with MS values of IVC were significantly higher than in healthy individuals, which suggests impaired vascular response to cold in the form of insufficient reduction of oscillation amplitudes of skin temperature. The index values for postcold vasodilation (IPV -1 and IPV -2 ) in group MS were significantly less due to the absence of an increase in oscillation amplitude of skin temperature to baseline values. We revealed a negative relationship of the values of the index of vasodilatation with marker ED-VEGF $(\mathrm{r}=-0.36, \mathrm{P}=0.008)$, In the MS group we found the correlation of an index of vasoconstriction with HOMA index $(\mathrm{r}=0.47, \mathrm{P}=0.004)$ and insulin $(\mathrm{r}=0.40, \mathrm{P}=0.003)$, therefore, the higher insulinemia and insulin resistance, the more disturbed vascular tone was detected.

We obtained a correlation between IPV -1 and some indicators of rhythmogram: the value of TR ( $\mathrm{r}=0.33, \mathrm{P}=0.02), \mathrm{SDNN}(\mathrm{r}=0.34, \mathrm{P}=0.01)$ and $\mathrm{CV} \%$ $(\mathrm{r}=0.42, \mathrm{P}=0.002)$. Insulin levels inversely correlated with an index of parasympathetic activity RMSSD ( $\mathrm{r}=$ $-0.28, \mathrm{P}=0.04)$. Therefore, the restoration of the amplitudes of the temperature fluctuations occurred after cold test. In other words, vasodilation depended on the higher variability of the heart rate.

\section{Discussion}

Currently, it is generally recognized that development of cardiovascular disease originates from endothelial dysfunction, however, pathogenetic mechanisms of its formation require further study. ${ }^{8} \mathrm{In}$ normal condition the joint functioning of the ANS and endothelium is aimed at ensuring the tone of the vascular wall: endothelium releasing the vasorelaxing biologically active substances, and vasoconstrictors from the sympathetic nerves. Balance between these opposing forces leads to the maintenance of vascular tone. ${ }^{9}$ Today, various methods for evaluation of en- dothelial damage were suggested; the most common are humoral markers, but the functional changes of the endothelium, usually preceded to morphological changes in the vascular wall. ${ }^{2}$ In our study, the functional condition of endothelium was assessed comprehensively considering the contents of the humoral markers of endothelin-1 and VEGF from serum and using WAST methods determining the response of vascular tone to the cold stimulus. The last method is an advanced, non-invasive technique, which confirmed their validity in clinical studies. ${ }^{7}$

It is known that obese patients with insulin-resistance have autonomic dysfunction, namely an imbalance between the activity of the sympathetic and parasympathetic divisions of the autonomic nervous system. ${ }^{2}$ This imbalance disrupts the adaptation to external influences and reinforces a pathological reaction. Food intake stimulates increased insulin levels, and it causes the activity of the sympathetic system, and in the obese patients it forms long-term hypersympathicotonia. ${ }^{10}$

Insulin-resistance that occurs during MS, leads to increased absorption of glucose in insulin-sensitive cells of the hypothalamus and disinhibits the sympathetic centers of the brain stem..$^{10}$ In addition, there is an increase in education angiotensinogen, which, through the increase of angiotensin- 2 also promotes activation of the sympathoadrenal system. ${ }^{3}$

Sympathetic nervous system can stimulate the release of reducing factors from the endothelium, such as endothelin-1. ${ }^{11,12}$ Its excessive concentration increases the sensitivity of vascular smooth muscle cells to noradrenaline, which leads to increased blood pressure and enhances vasoconstrictor responses to sympathetic stimulation. ${ }^{5,13}$ Thus, in MS there are conditions for disturbances of autonomic innervation and the formation of endothelial dysfunction.

The results of our study indicate hypersympathicotonia in patients with MS. Similar data were obtained in groups of patients with diabetes type 2 and obesity. ${ }^{14,15}$ However, when conducting an active orthostatic probe there is no adequate increase in the sympathetic

Table 4. Indices of vasoconstriction and post-cold vasodilation.

\begin{tabular}{lcccc}
\hline Range of oscillations & Variant & IVC & IPV $-\mathbf{1}$ & IPV $-\mathbf{2}$ \\
\hline Endothelial & Controls & $0.47 \pm 0.19$ & $1.11 \pm 0.51$ & $1.03 \pm 0.49$ \\
\cline { 2 - 5 } & $\mathrm{MS}$ & $0.62 \pm 0.25^{*}$ & $0.88 \pm 0.53^{*}$ & $0.71 \pm 0.35^{*}$ \\
\hline Neurogenic & Controls & $0.43 \pm 0.23$ & $0.95 \pm 0.44$ & $0.9 \pm 0.25$ \\
\cline { 2 - 5 } & $\mathrm{MS}$ & $0.70 \pm 0.68^{*}$ & $1.0 \pm 0.73$ & $0.82 \pm 0.53$ \\
\hline Myogenic & Controls & $0.47 \pm 0.23$ & $0.75 \pm 0.34$ & $0.71 \pm 0.18$ \\
\cline { 2 - 5 } & MS & $0.64 \pm 0.36$ & $0.90 \pm 0.38$ & $0.93 \pm 1.15$ \\
\hline
\end{tabular}

IVC, index of vasoconstriction; IPV, index post-cold vasodilation. ${ }^{*} \mathrm{P}<0.05$ between the groups, in the appropriate ranges. 
component, which is a sign of decrease of the heart rate variability. These characteristics also include a decrease in the total power spectrum, declines in SDNN in MS, which were confirmed in other studies. ${ }^{16}$

In patients with MS microvascular response to cold stress was lower than in healthy individuals and was not restored after cold exposure. The lack of vasodilator phase in patients with MS may be evidence for the presence of vasomotor form of endothelial dysfunction. In persons with MS, the contents of endothelin-1 and VEGF in the blood plasma are significantly higher than the level of the comparison group, which characterizes the existing endothelial dysfunction. The increase in humoral markers of endothelial damage (VEGF and ET-1) was consistent with functional changes in cold test. Thus, MS is marked with broken vasodilatory response due to the release of humoral vasoconstrictors. It was also found that the violation of the functional state of the endothelium significantly correlated with indicators reflecting the reduction of heart rate variability. Thus, in metabolic syndrome we found impaired endothelial function associated with decrease of heart rate variability.

\section{Conclusions}

Endothelial dysfunction in metabolic syndrome is manifested by increased biochemical markers: VEGF, ET-1. When carrying out the methods of wavelet analysis of skin temperature oscillations with cold breakdown, we revealed the failure vasodilatory mechanisms of regulation of vascular tone, correlated with laboratory indices of endothelial dysfunction.

Patients with metabolic syndrome are characterized by reduced heart rate variability in the background state and during the orthostatic test. The severity of disorders of autonomic regulation is associated with the degree of endothelial dysfunction.

\section{References}

1. Campbell B, Aguilar M, Bhuket T, et al. Females, Hispanics and older individuals are at greatest risk of developing metabolic syndrome in the U.S. Diabetes Metab Syndr 2016 [Epub ahead of print].
2. Patel TP, Rawal K, Bagchi AK, et al. Insulin resistance: an additional risk factor in the pathogenesis of cardiovascular disease in type 2 diabetes. Heart Fail Rev 2016;21:11-23.

3. Gurgenian SV, Vatinian SKh, Zelveian PA. Arterial hypertension in metabolic syndrome: pathophysiological aspects. Ter Arkh 2014;86:128-32.

4. Saladini F, Di Marco A, Palatini P. Autonomic dysfunction: how to identify and when to treat? High Blood Press Cardiovasc Prev 2016 [Epub ahead of print].

5. Bruno RM, Ghiadoni L, Seravalle G, et al. Sympathetic regulation of vascular function in health and disease. Front Physiol 2012;3:284.

6. Rajendran P, Rengarajan T, Thangavel J, et al. The vascular endothelium and human diseases. Int J Biol Sci 2013;9:1057-69.

7. Smirnova E, Podtaev S, Mizeva I, Loran E. Assessment of endothelial dysfunction in patients with impaired glucose tolerance during a cold pressor test. Diabet Vasc Dis Res 2013;8:1-9.

8. Cubbon RM, Kahn MB, Wheatcroft SB. Effects of insulin resistance on endothelial progenitor cells and vascular repair. Clin Sci 2009;117:173-90.

9. Favero G, Paganelli C, Buffoli B, et al. Endothelium and its alterations in cardiovascular diseases: life style. Intervent Biomed Res Int 2014;801896.

10. do Carmo JM, da Silva AA, Wang Z, et al. Obesity-induced hypertension: brain signaling pathways. Curr Hypertens Rep 2016;18:58.

11. Barton M, Baretella O, Meyer MR. Obesity and risk of vascular disease: importance of endothelium-dependent vasoconstriction. Br J Pharmacol 2012;165:591-602.

12. Lobato NS, Filgueira FP, Akamine EH, et al. Mechanisms of endothelial dysfunction in obesity-associated hypertension. Braz J Med Biol Res 2012;45:392-400.

13. Franz R, Maturana MA, Magalhães JA, et al. Central adiposity and decreased heart rate variability in postmenopause: a cross-sectional study. Climacteric 2013; 16:576-83.

14. Wu HT, Hsu PC, Sun CK, et al. Assessment of autonomic dysfunction in patients with type 2 diabetes using reactive hyperemia. J Theor Biol 2013;330:9-17.

15. Indumathy J, Pal GK, Pal P, et al. Association of sympathovagal imbalance with obesity indices, and abnormal metabolic biomarkers and cardiovascular parameters. Obes Res Clin Pract 2015;9:55-66.

16. Gaudreault V, Després JP, Rhéaume C, et al. Exerciseinduced exaggerated blood pressure response in men with the metabolic syndrome: the role of the autonomous nervous system. Blood Press Monit 2013; 18:252-8. 